
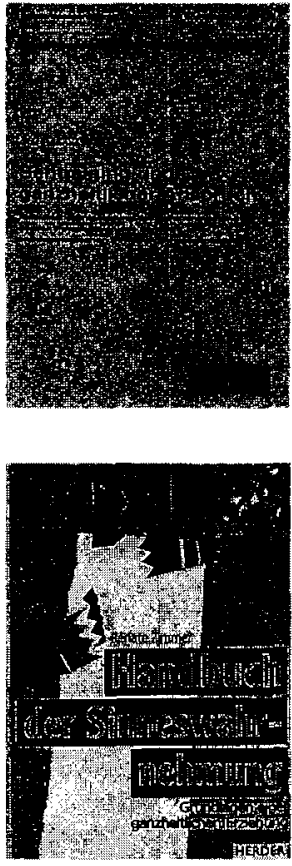

OLAF HÖHNKE:

\section{Sehtraining und ganzheitlicher Anspruch}

Historische Entwicklung des Sehtrainings und dessen Perspektiven aus der Sicht einer anthropologischen Bewegungswissenschaft

(Sportwissenschaftliche Dissertationen und Habilitationen. Bd. 32).

Hamburg: Czwalina-Verlag 1994. 299 S.; DM 40,-

RENATE ZIMMER:

\section{Handbuch der Sinneswahrnehmung}

Grundlagen einer ganzheitlichen Erziehung

Freiburg i. Br.: Verlag Herder 1995. 216 S., DM 34,-
„Mit allen Sinnen leben“"zu lehren, die Sinne zu „schärfen“ und ihren Gebrauch zu schulen - das ist ein Anliegen der Leibeserziehung mit langer Tradition. Übungen unter diesem Anspruch finden sich z. B. schon bei den Philanthropen am Ende des 18. Jh.s, später im deutschen Turnen. Die Schulung der Wahrnehmung, insbesondere das Eingehen auf Wahrnehmungsschwächen, ist auch ein geläufiger Aspekt der psychomotorischen Erziehung. Seit einigen Jahren ist er im Zusammenhang mit dem Programm der „Körpererfahrung“, besonders von FUNKE und TREUTLEIN, aufgegriffen worden. Grundlage dieser und anderer praktisch pädagogischer Traditionen ist die Erkenntnis vom engen Zusammenhang oder sogar der „Einheit" (V. VON WEIZSÄCKER) von Bewegung und Wahrnehmung: Kinder erschließen sich ihre Welt durch Bewegung; Bewegungen zu erlernen oder an veränderte Umgebungs- bedingungen anzupassen ist immer auch mit Anforderungen an die Wahrnehmung verbunden.

In der sportwissenschaftlichen Forschung hat der Zusammenhang zwischen Bewegung und Wahrnehmung jedoch bisher eher in der anderen Richtung Interesse gefunden: Wer Bewegungen optimieren möchte, insbesondere im Leistungssport, muB oft auch die Wahrnehmung verbessern. Sportliches Training schlieBt Wahrnehmungstraining ein. Welche Wahrnehmungssysteme in dieser Hinsicht von $\mathrm{Be}$ deutung sind und wie ihre Leistungsfähigkeit erhöht, ja: trainiert werden kann, ist daher ein Thema in der Bewegungs- und Trainingsforschung. Arbeiten wie die MESTERs (1988) und ENNENBACHs (1991) lassen erkennen, daß solche Forschungsergebnisse über ihre trainingspraktische Bedeutung hinaus auch helfen können, das Grundlagenwissen über die menschliche 